\title{
Silicates as fillers for polymer composites
}

\author{
Oleksandra Dzeikala $^{1}$, dr Mirosława Prochon $^{1}$ \\ 1. Institute of Polymer and Dye Technology, University of \\ Technology, POLAND, Lodz, Stefanowskiego 12/16, 90-924, E-mail: Dzeikala.sandra@ gmail.com \\ 2. Institute of Polymer and Dye Technology, University of \\ Technology, POLAND, Lodz, Stefanowskiego 12/16, 90-924,, E-mail: miroslawa.prochon@ @.lodz.pl
}

\begin{abstract}
Polyethylene is widely used in the packaging industry and is characterized by high mechanical strength and high chemical resistance. New properties can be obtained through the use of fillers such as layered silicate. In this study, a filler was added to the composite with a size of an hour ontice ranging from 63 um to $125 \mu m$.
\end{abstract}

Polyolefins, polyethylene, filler, mineral fillers, vermiculite, packaging

\section{Modification of polyethylene}

Polyethylene is characterized by high elasticity of C-C bonds, and in combination with low intermolecular interaction of chains, this polymer has a relatively low melting point. Depending on the preparation method, the polymer can have a linear or highly branched structure. The main products obtained from various types of polyethylene are packaging materials, and due to good biocompatibility and chemical inactivity, it is widely used in medical devices: filters, bottles and disposable ampoules. [1]

Polyolefins, including polypropylene, are a group of polymers used in mass production areas, and due to their average physicochemical properties (mainly low melting point and not very high tensile strength), they are not used as construction materials. [1]

However, the list of fillers modifying the properties of the material is surprisingly extensive. It includes both the simplest inorganic fillers and polyhedral oligomeric silesquioxanes (POSS). Among the fillers, the following are distinguished: calcium carbonate; talc; silica; glass flour; nanocellulose; cellulose fillers.

Regardless of the application, plastics require special protection against the risk of fire. Almost most plastics are not very resistant to heat and direct fire, including polymers from the group of polyolefins: polyethylene or polypropylene. Some types of plastics, during their thermal decomposition, emit poisonous, irritating or extremely harmful chemical compounds, including epichlorhydrin, vinyl chloride, $\mathrm{HCl}$ or hydrogen cyanide. [2]

Analyzing the research conducted so far, it turns out that vermiculite can be a filler that works effectively as a flame retardant. Its presence in the polymer matrix causes that the combustion process is less rapid compared to the reference, unfilled samples. It also turns out that in situ expanded vermiculin can compete with its flame retardant properties with commercially available inorganic fillers - sepiolite or smectite.

\section{References}

[1] Moosavi, A. N. (2003). Fusion-Bonded Epoxy (FBE): A Foundation for Pipeline Corrosion Protection. Corrosion 59, 12.

[2] Riegert D. (2013). The ability to modification the properties of flammable plastics (7th ed.). BADANIA I ROZWÓJ, 30(2), https://panel.sft.cnbop.pl/storage/91dad163-497e-49409078-f3538e291666 